\title{
Éditorial: \\ Énoncé de politique sur une approche individualisée à la promotion de la santé des personnes âgées
}

Énoncé du problème: La promotion de la santé que l'on définit actuellement comme «le processus permettant aux individus et aux communautés de mieux contrôler et gérer leur santé» (traduction libre) (Charte d'Ottawa, Epp, 1986; OMS, 1984, p. 3) présente des défis particuliers à ceux qui participent aux politiques, à la planification et à la prestation des services de santé destinés aux personnes âgées. L'un de ces défis tient au fait que plus de 80 pour cent de personnes âgées souffrent de maladies chroniques (Conseil consultatif national sur le troisième âge, 1989). Les invalidités fonctionnelles et les maladies des aînés ${ }^{1}$ sont souvent traitées comme des affections aiguës et non comme des problèmes persistants qui nécessitent un engagement constant en faveur de la promotion de la santé et de la prévention de la maladie. C'est pourquoi les personnes âgées deviennent facilement des «patients» qui dépendent des professionnels pour gérer leur santé. Cette relation se traduit par une dévalorisation et un manque de confiance en soi qu'intensifient encore la discrimination fondée sur l'âge et les rapports de forces asymétriques entre personnes âgées et soignants professionnels (McWilliam, Belle Brown, Carmichael, \& Lehman, 1994).

Portée: Près de 80 pour cent des personnes âgées se font aider pour accomplir au moins une activité, y compris toutes sortes d'activités essentielles de la vie de tous les jours (Conseil consultatif national sur le troisième âge, 1993), pourtant seules 20 pour cent d'entre elles disent être très limitées dans leur autonomie et 64 pour cent déclarent que leur santé est «bonne» à «excellente» pour leur âge (Conseil consultatif national sur le troisième âge, 1993). La grande majorité des personnes âgées souffrant de maladie chronique ont la détermination morale (Conseil consultatif national sur le troisième âge, 1990), les capacités mentales (Baltes, Kliegl, \& Dittman-Kohli, 1988) et la motivation (Benson et al., 1989; Hall et al., 1992; Higgins, 1989) nécessaires pour jouer un rôle proactif dans la promotion de leur santé.

Analyse des résultats d'études: On a constaté que, chez les personnes âgées, le fait de s'adonner à des activités de promotion de la santé améliorait les résultats de santé (Kaplan, Greenfield, \& Ware, 1989), le sentiment de maîtriser sa vie et d'être autonome (Berkowitz, Waxman, \& Yaffe, 1988), l'image de soi et, donc, l'autocontrôle (Braden, 1990; Smits \& Kee, 1992), 
ainsi que la qualité de vie (Smits \& Kee, 1992). On a associé la prise en charge personnelle à la vigueur intellectuelle, psychologique, comportementale et physiologique (Spinhoven, Ter juile, Linssen, \& Gazendam 1989). Ces conclusions suggèrent que les modèles de soins visant à encourager les individus à jouer un rôle proactif dans la promotion de la santé et la prévention de la maladie sont une composante importante des services de santé pour personnes âgées. En effet, nombre d'activités de promotion de la santé ont permis d'améliorer considérablement les taux de mortalité, l'autonomie fonctionnelle, les mesures du bien-être, la qualité de vie, l'auto-efficacité et les pratiques de santé (Leigh et al., 1992; Fries, Bloch, Harrington, Richardson, \& Beck, 1993; Elder, Williams, \& Drew, 1995; Ruffing-Rahal, 1994; Cox and Parsons, 1996; McWilliam et al., 1999; Schweitzer et al., 1994; Hall et al., 1992; Hamdorf, Withers, Penhall, \& Haslam, 1992).

Recommandations: L'Association canadienne de gérontologie rejette le mythe sociétal qui veut que la promotion proactive de la santé parmi les personnes âgées n'ait aucun effet sur les intéressés et pense, au contraire, que la promotion de la santé est une priorité valable, même pour les personnes frêles et souffrant de maladie chronique.

L'Association canadienne de gérontologie soutient les efforts pour faire mieux valoir l'importance d'adopter une approche individualisée en matière de promotion de la santé et de soins préventifs pour les personnes âgées, et d'intégrer la promotion de la santé aux soins traditionnels axés sur la maladie.

L'Association canadienne de gérontologie appuie le financement public des activités de promotion de la santé et de prévention de la maladie s'adressant aux personnes âgées.

L'Association canadienne de gérontologie recommande que les établissements d'enseignement supérieur offrent davantage de cours sur la promotion de la santé individualisée et ses applications cliniques dans tous les domaines des sciences de la santé et du service social.

\section{Notes}

1 Le masculin embrasse le féminin.

L'Association canadienne de gérontologie reconnaît aussi que les politiques de santé doivent bénéficier de l'aide de la communauté et s'appuyer sur le modèle de gestion de la santé de la population.

\section{Références}

Baltes, P.B., Kliegl, R., \& Dittman-Kohli, F. (1988). On the locus of training gains in research on the plasticity of fluid intelligence in old age. Journal of Educational Psychology, 880, 392-400. 
Benson, L., Nelson, E., Napps, S., Roberts, E., Kane-Williams, E., \& Salisbury, R. (1989). Evaluation of staying healthy after fifty educational program: Impact on course participants. Health Education Quarterly, 16(4), 485-508.

Berkowitz, M.W., Waxman, R., \& Yaffe, L. (1988). The effects of a resident self-help model on control, social involvement and self-esteem among the elderly. The Gerontologist, 28(5), 620-624.

Braden, C.J. (1990). A test of the self-help model: Learned response to chronic illness experience. Nursing Research, 39(1),42-47.

Conseil consultatif national sur le troisième âge. (1989). Understanding seniors' independence report \#1: The barriers and suggestions for action. Ottawa: Ministère des Approvisionnement et des Services Canada.

Conseil consultatif national sur le troisième âge. (1990). A portrait of seniors in Canada. Ottawa: Statistique Canada, cat. no. 89-159.

Conseil consultatif national sur le troisième âge. (1993). Aging vignettes. Ottawa: Gouvernement du Canada.

Cox, E.O., \& Parsons, R.R. (1996). Empowerment-oriented social work practice: Impact of late life relationships of women. Journal of Women and Aging, 8, $129-143$.

Elder, J.P., Williams, S.J., \& Drew, J.A. (1995). Longitudinal effects of preventive services on health behaviors among an elderly cohort. American Journal of Preventive Medicine, 11, 354.

Epp, J. (1986). La santé pour tous: plan d'ensemble pour la promotion de la santé. Ottawa: Ministre des Approvisionnements et des Services.

Fries, J.F., Bloch, D.A., Harrington, H., Richardson, N., \& Beck, R. (1993). Twoyear results of a randomized controlled trial of a health promotion program in a retiree population: The Bank of America study. The American Journal of Medicine, 94, 455-462.

Hall, N., De Beck, P., Johnson, D., Mackinnon, K., Gutman, G., \& Glick, N. (1992). Randomized trial of a health promotion program for frail elders. La Revue canadienne du vieillissement, 11, 72-100.

Hamdorf, P.A., Withers, R.T., Penhall, R.K., \& Haslam, M.V. (1992). Physical training effects on the fitness and habitual activity patterns of elderly women. Archives of Physical Medicine and Rehabilitation, 73, 603-608.

Higgins, P. (1989). Short term changes in health behaviours of older adults. The Canadian Journal of Nursing Research, 21(1), 19-32.

Kaplan, G.A., Greenfield, S., \& Ware, J.E. Jr. (1989). Assessing the effects of physician-patient interactions on the outcomes of chronic disease. In K.N. Lohr (Ed.), Advances in health status assessment. Medical Care, 27(suppl. 1), 110-127.

Leigh, J.P., Richardson, N., Beck, R., Kerr, C., Harrington, H., Parcell, C.L., \& Fries, J.F. (1992). Randomized controlled study of a retiree health promotion program: The Bank of America study. Archives of Internal Medicine, 152, 1201-1206.

McWilliam, C.L., Belle Brown, J., Carmichael, J.L., \& Lehman, J.M. (1994). A new perspective on threatened autonomy in elderly persons: The disempowering process. Social Science and Medicine, 38, 327-338.

McWilliam, C.L., Stewart, M., Brown, J.B., McNair, S., Donner, A., Desai, K., Coderre, P., \& Galajda, J. (1999). A randomized control trial of a critical reflection approach to home-based health promotion for chronically ill older persons. Health Promotion International, 14(1), 27-41. 
Organisation mondiale de la santé (OMS). (1984). Health promotion, a discussion document on the concept and principles. Copenhagen; OMS, Bureau régional pour l'Europe.

Ruffing-Rahal, M.A. (1994). Evaluation of group health promotion with community-dwelling older women. Public Health Nursing, 11, 38-48.

Smits, M., \& Kee, C. (1992). Correlates of self-care among the independent elderly: Self-concept affects well-being. Journal of Gerontological Nursing, 18(9), 13-18.

Spinhoven, P., Ter juile, M., Linssen, A., \& Gazendam, B. (1989). Pain coping strategies in a Dutch population of chronic low back pain patients. Pain, 37(1),77-83.

Schweitzer, S.O., Atchison, K.A., Lubben, J.E., Mayer-Oakes, S.A., De Jong, F.J., \& Matthias, R.E. (1994). Health promotion and disease prevention for older adults: Opportunity for change of preaching to the converted? American Journal of Preventive Medicine, 10, 223-229.

Rédigé par Carol McWilliam, Ed.D., à la demande de conseil d'administration de l'Association canadienne de gérontologie, l'exposé ci-dessus constitute la position officielle du conseil adoptée le 26 octobre 2000. Cet énoncé est basé sur l'article "Care Delivery Approaches and Seniors' Independence" par C.L. McWilliam, W.L. Diehl-Jones, J. Jutai, et S. Tadrissi (La Revue canadienne du vieillissement (2000), 19(suppl. 1), 101-124.). 